\title{
Sentidos y formas de participar en la escuela: la perspectiva de los jóvenes argentinos
}

Meanings and ways of participation in school: the perspective of youth Argentinians

\author{
Volumen 18, Número 1 \\ Enero-Abril \\ pp. 1-20
}

Este número se publica el $1^{\circ}$ de enero de 2018

DOI: https://doi.org/10.15517/aie.v18i1.31810

Julia Lucas

Revista indizada en REDALYC, SCIELO

Revista distribuida en las bases de datos:

LATINDEX, DOAJ, REDIB, IRESIE, CLASE, DIALNET, SHERPA/ROMEO, QUALIS-CAPES, MIAR

Revista registrada en los directorios:

ULRICH'S $, \underline{R E D I E}, \underline{R I N A C E}, \underline{\text { OEI }}, \underline{\text { MAESTROTECA }}, \underline{\text { PREAL, }} \underline{\text { CLACSO }}$ 


\title{
Sentidos y formas de participar en la escuela: la perspectiva de los jóvenes argentinos \\ Meanings and ways of participation in school: the perspective of youth Argentinians
}

\begin{abstract}
Julia Lucas ${ }^{1}$
Resumen: Promover la participación de los más jóvenes, tanto dentro como fuera de la escuela, ha devenido, en los últimos tiempos, en objeto de especial atención en Argentina. Ello se logra haciendo visible en la implementación de diversas líneas, programas y proyectos especialmente gubernamentales. A partir de la perspectiva situacional del aprendizaje, este artículo describe y analiza los sentidos y las significaciones que los jóvenes estudiantes del nivel secundario asignan a sus prácticas de participación en diversos espacios curriculares. Para ello, y a partir de una perspectiva de tipo etnográfica, esta investigación se realizó en un curso de cuarto año de una escuela secundaria, situada en la Ciudad de Quilmes, Provincia de Buenos Aires. Se realizaron observaciones participantes, entrevistas a estudiantes de entre 17 y 18 años de edad, docentes y directivos. Se aplicaron técnicas, tales como la reflexión de los estudiantes acerca de filmaciones de sus propias clases, así como escritura analítica respecto de su participación al terminar cada una de las clases. Estas primeras aproximaciones dan cuenta de que "participar" implica, para estos estudiantes, satisfacer principalmente las expectativas de sus profesores, así como cumplir con las normas que rigen la vida en el aula. Ser aprobado o no, se convierte en el motor de las acciones en la vida cotidiana, aun cuando ir a la escuela cobre un sentido trascendental para estos jóvenes. Se concluye que si las acciones en el aula se vinculan con actuar, según el deber ser, habría que reflexionar en qué lugar queda aquello que acontece con el aprendizaje.
\end{abstract}

Palabras clave: jóvenes, educación secundaria, aprendizaje, participación estudiantil.

Abstract: In recent years, the promotion of young people practices inside and outside the boundaries of school has become an object of special attention in Argentina, made visible thru implementation of diverse research lines, programs and projects, mainly in the governmental sphere. From a situational learning perspective, this article describes and analyzes meanings and significances that young students from secondary School give to their practices of participation in diverse curricular spaces. For that purpose, and based on an ethnographic approach, this research was developed on a fourth grade course of a Secondary School, located in Quilmes City, Province of Buenos Aires. Participant Observation and interviews were made to students between 17 and 18 years old, also to teachers and managerial staff. Diverse techniques, like students' reflections about filming of their own courses and reflective writing about their participation at the end of every course, were applied. These initial approaches show that "participating" implies for these students mainly to satisfy and to accomplish teachers' expectations and norms that lead life in classrooms. Therefore, to be or not to be approved becomes the motor of actions of daily life, even if going to School has a transcendental meaning for this youngsters. It is concluded that if actions in classroom are tied according to what "ought to be", then We must think about in which place stands all that happens in learning.

Keywords: young people, secondary education, learning, student participation

\footnotetext{
1 Docente e investigadora de la Universidad Nacional de Quilmes (UNQ) y la Universidad Pedagógica Nacional (UNIPE), Argentina. Maestranda en Antropología Social (IDES/IDAES/UNSAM), Argentina.
}

Dirección electrónica: juliaplucas@gmail.com

Artículo recibido: 4 de abril, 2017

Enviado a corrección: 12 de setiembre, 2017

Aprobado: 13 de noviembre, 2017 


\section{Introducción}

La participación de los jóvenes en diferentes prácticas sociales y culturales parece haber concentrado, en las últimas décadas, la atención de una serie de diversos sectores sociales, políticos y culturales. Solo para dar cuenta de algunos de ellos, se puede señalar la importancia adjudicada a la participación política de esta población por parte de los movimientos y de las organizaciones sociales, así como por parte de los partidos políticos. El ámbito académico, especialmente el ámbito de las ciencias sociales, ha destinado en los últimos tiempos buena parte de sus esfuerzos a problematizar aquellos discursos que aluden a la supuesta apatía o desinterés de los jóvenes por lo político, lo cual contribuye a echar luz sobre las formas en que las poblaciones infantiles y juveniles participan de los procesos de movilización social. Finalmente, cabe señalar que han logrado poner de relieve la necesidad de discutir acerca de qué se entiende por política cuando de prácticas infantiles y juveniles se trata (Lucas, 2015).

Por su parte, desde las esferas gubernamentales, se promovió, en los últimos años, una serie de diversos programas y proyectos destinados a promover mayores grados de participación en la niñez y la juventud ${ }^{2}$, siendo el territorio educativo un espacio propicio para la puesta en marcha de este tipo de política. Por último, no se puede desestimar el papel desempeñado por los medios de comunicación -y del sentido común- a la hora de venerar, demonizar o criminalizar a los jóvenes y/o a sus prácticas de participación en la vida pública.

En este contexto, es posible señalar que las prácticas de participación de los más jóvenes devienen en un objeto donde se disputan una serie de diversos sentidos y significaciones que no eximen al territorio escolar, sino que lo interpelan. En relación con ello, es importante señalar la existencia de investigaciones donde se advierten algunas de las tensiones existentes entre la necesidad de promover mayores niveles de participación y autonomía en el estudiantado dentro de la escuela, y la naturalidad con la que se asume el dispositivo escolar. La a-historicidad con la se concibe el espacio escolar - y sus formasimpide visibilizar el modo en que la niñez y la juventud son infantilizados dentro de dicho espacio.

Esto se ve con mucha claridad en aquellos niños y jóvenes que fuera de los muros escolares ocupan lugares de responsabilidad, o que se manejan con más autonomía que en la escuela, pero que dentro de ella son tratados bajo las premisas de la heteronomía y la

\footnotetext{
2 Se pueden mencionar aquí, Programas y/o Proyectos tales como: "Formación Ciudadana", "Organización Popular", "Participación Solidaria". Enmarcados en la Dirección Nacional de la Juventud (DINAJU) del Ministerio de Desarrollo Social de la Nación, los cuales muchas veces trabajan en conexión con las escuelas (Lucas, 2015).
} 
obediencia (Kaplún, 2004; Batallán y Campanini, 2008). Asimismo, otra de las tensiones presentadas desde algunas investigaciones se relacionan con haber detectado cierta concepción donde la imagen del "Buen estudiante" es equiparada al "Estudiante obediente" o al "Estudiante brillante", como consecuencia se omite la problematización acerca de las potencialidades de los sujetos para construir aprendizajes críticos y reflexivos (Kravetz y Castro, 2015).

En este orden de problematizaciones, la investigación donde se enmarca este trabajo se preguntó particularmente respecto de las formas en que los y las estudiantes participan en sus experiencias escolares, así como por los sentidos y las significaciones que los y las jóvenes adjudican a sus formas de participar. Desde una perspectiva de tipo etnográfica, este estudio se realizó en una escuela secundaria del Distrito de Quilmes, Provincia de Buenos Aires, Argentina, con jóvenes de entre 16 y 17 años de edad. El carácter polisémico del término participación fue adquiriendo definiciones, que esta vez no vendrían dadas por los adultos, ni académicos, sino que fue develando sus matices a partir de las voces y las perspectivas de los mismos jóvenes estudiantes.

\section{Referencias teóricas}

\subsection{Prácticas de participación en la escuela}

En el ámbito educativo, la preocupación por profundizar las prácticas de participación del estudiantado se tradujo en la elaboración y ejecución de una serie de iniciativas provenientes de diversos sectores y niveles del sistema educativo. Así, por ejemplo, desde el ámbito gubernamental, especialmente entre el período que comprende los años 2003 y 2015, se visibilizó la aparición de proyectos y programas ${ }^{3}$ enmarcados en una política educativa que señalaba la necesidad de profundizar la democratización de las instituciones educativas ${ }^{4}$.

No obstante, muchas veces son las mismas escuelas las que han intentado -e intentan- vincularse con otras organizaciones o movimientos, tanto sociales como políticos,

\footnotetext{
${ }^{3}$ Se hace referencia, por ejemplo, al proyecto "Parlamento Juvenil del MERCOSUR" de la Dirección Nacional de Políticas Socio -Educativas del Ministerio de Educación Nacional, el Programa "Construcción de ciudadanía en las escuelas" del Ministerio de Educación de la Nación, el "Programa Centro de Actividades Juveniles" de la Dirección General de Cultura y Educación de la Provincia de Buenos Aires. Estas son algunas de las iniciativas gubernamentales destinadas al ámbito escolar, preocupadas por promover allí la participación ciudadana de los jóvenes (Lucas, 2015).

${ }^{4}$ Ver más en: Ministerio de Educación de la Nación (2015) Miradas en torno a la democratización de la escuela secundaria: Aportes y desafíos. Ciudad Autónoma de Buenos Aires. ISBN 978-950-00-1149-5. Versión web: http://entrama.educacion.gov.ar/uploads/secundaria-para todos/Miradas Entorno a la Democratizacion.pdf
} 
como una forma posible de acercar la experiencia escolar a la realidad social, o al revés, dejando que las comunidades entren a la escuela. En este sentido, cabe señalar que muchos docentes vienen siendo, desde hace décadas, promotores de experiencias cuyo objetivo ha sido generar prácticas democráticas concretas - y no de mera enunciación- en la escuela (Redondo, 2004). Como se ha señalado en otro ámbito (Baquero y Lucas, 2015), muchos de estos docentes suelen asignar a su trabajo cierto sentido político o social, y es desde allí donde se posicionan a la hora de habilitar dichas experiencias. Para otros docentes, promover una mayor participación de los estudiantes se encuentra relacionado con el objetivo de mejorar los aprendizajes o volverlos más significativos (Barquero y Lucas, 2015.

Esta breve pincelada, por las diversas iniciativas, no pretende agotar la descripción de las experiencias existentes, lo que sí busca es poner de relieve la importancia que ha venido teniendo la necesidad de pensar las prácticas de participación del estudiantado en sus experiencias educativas.

En resumen, ya sea como parte de la gestación de una política educativa o como iniciativa de las propias escuelas y/o de sus docentes, que los estudiantes participen y se apropien de sus experiencias escolares es desde hace tiempo una preocupación común. No obstante, y pese a los diversos esfuerzos por promover mayores grados de participación de los estudiantes, lo que parece haber de base es una cierta invisibilización y naturalización del espacio escolar, una cierta invisibilización de los sentidos y las formas que lo escolar adoptó en la modernidad, y en virtud de esto, una cierta forma de tramitar las prácticas de enseñanza y el aprendizaje en el aula.

En cuanto a los sentidos, cabe recordar, que la escuela nace para forjar a los hombres de la Modernidad y de este modo, dar lugar a la realización de la humanidad, o en otro nivel de análisis, para introducir a los sujetos en una particular forma de vida democrática, para forjar un cierto tipo de ciudadano, el ciudadano de los Estados Nación.

En cuanto a las características de la experiencia escolar moderna, resulta importante, que ella parece conservar aún componentes nodales, tales como la gradualidad, la simultaneidad sistemática y cierta particularidad en la distribución de posiciones subjetivas de docentes y estudiantes. En cuanto a la gradualidad, se puede decir que la escuela moderna se ha organizado con base en el trípode edad/grado académico/contenidos curriculares. Las trayectorias educativas de los sujetos debieron $-\mathrm{y}$ deben- ajustarse al recorrido -universal y homogéneo- propuesto desde la escuela. 
La simultaneidad sistémica, por su parte, ha implicado una organización donde un docente enseña a un grupo numeroso de estudiantes de manera simultánea. Es decir, los mismos contenidos al mismo tiempo para todos los estudiantes. Finalmente, la clásica distribución de posiciones subjetivos, en el marco del formato escolar moderno, establece una relación donde es el docente el que posee el saber, mientras el alumno permanece en condición de ignorante (Ranciere, 2007).

El aumento en la participación y en la autonomía de los jóvenes en sus prácticas cotidianas, y especialmente en sus prácticas educativas parecen ponerse en tensión con la permanencia de buena parte de las características de la experiencia escolar moderna descriptas en los párrafos anteriores. Esto es sumamente inquietante en un contexto donde se reconoce el grado de autonomía que niños y jóvenes han ganado en sus contextos cotidianos, sus posibilidades de acceder a diversos conocimientos con mucha facilidad o los lugares de responsabilidad que asumen en sus contextos familiares, locales o comunitarios. En este sentido, recuerda Baquero (2001) que el rol de estudiante se ha definido en relación con las características de la infancia moderna, y esto implica que el formato escolar en que se organiza la escuela moderna establece para los estudiantes una relación heterónoma respecto a los docentes, y una incorporación graduada a la cultura escolar y adulta. Lo que se delata, en definitiva, es un grado importante de desencuentro entre las culturas infantiles y juveniles y la cultura escolar (Lucas, 2015).

En resumen, en un contexto donde, desde diferentes sectores y actores, se busca promover la participación de los más jóvenes, y una escuela cuyos fines y formas suele mostrarse desencontrada respecto de las culturas juveniles, nos preguntamos qué sucede con la participación de los jóvenes en sus escuelas o, más concretamente, qué sucede con la participación en los diferentes espacios curriculares por los cuales transitan cotidianamente. Nos interesa particularmente responder estas preguntas a partir de las voces y perspectivas de los mismos estudiantes.

\section{Metodología}

A partir de la realización de un proyecto de investigación denominado "La inclusión de las voces de los estudiantes en los procesos de aprendizaje. Variaciones de las formas de 
participación y apropiación de la experiencia escolar en el nivel medio" ${ }^{5}$, cuyo objetivo fue explorar las formas de participación de estudiantes del nivel secundario en proyectos y experiencias educativas preocupadas por promover formas alternativas de participación, tanto en el marco de sus experiencias escolares como en la gestión de sus aprendizajes, esta investigación se propuso indagar los sentidos y significaciones que adquiere, para los estudiantes, participación en sus experiencias escolares.

Según una investigación basada en el método etnográfico, se abordó la experiencia de un grupo de estudiantes de cuarto año de la escuela secundaria ${ }^{6}$ ubicada en la localidad de San Francisco Solano, Partido de Quilmes, Provincia de Buenos Aires, Argentina. Se realizaron allí observaciones participantes, entrevistas en profundidad, grupos focales y registros de clase hechos por los mismos estudiantes. Se filmaron clases para luego usar el material en los grupos focales, de modo que los estudiantes pudieran reflexionar sobre sus formas de participación en clase ${ }^{7}$. Se entrevistaron docentes, preceptores, estudiantes y equipos directivos.

Las indagaciones se relacionaron con poder registrar y reflexionar no solo respecto de las formas de participar en cada uno de los espacios curriculares, sino también con poder indagar qué entienden los diversos actores por participación, y que piensan -especialmente los estudiantes - respecto de sus formas de participar en las escuelas. Se abordaron cinco (5) espacios curriculares enmarcados en el diseño curricular oficial para el nivel. Ellos son Política y Ciudadanía, Sistemas de Información Contable (SIC), Inglés, Geografía y Gestión de las Organizaciones. Asimismo, se observaron actividades relacionadas con la conformación del Centro de Estudiantes, así como las actividades realizadas en el marco de un proyecto denominado NEPSO (Nuestra Escuela Pregunta Su Opinión), desarrollado en el marco de la materia Gestión de las Organizaciones.

El proyecto Nepso promueve la realización de investigaciones desde la escuela. Los docentes son convocados a coordinar dichos proyectos, pero el tema de investigación y la investigación misma son llevados adelante por el estudiantado.

La observación de la conformación del Centro de Estudiantes se relacionó con el hecho de que allí el grupo de estudiantes de toda la escuela debía presentar propuestas

\footnotetext{
5 PICT 2194E1293/12 "La inclusión de las voces de los estudiantes en los procesos de aprendizaje. Variaciones de las formas de participación y apropiación de la experiencia escolar en el nivel medio", Dirigido por Ricardo Baquero. Universidad Nacional de Quilmes. Argentina.

${ }^{6}$ En Argentina, la estructura de la escuela secundaria se encuentra organizada en seis años.

7 La publicación de las voces de los diversos actores educativos fue autorizada por ellos mismos. No obstante, la referencia a dichas voces guarda absoluta confidencialidad.
} 
diseñadas los y las estudiantes, el ganador sería el grupo que contara con mayor cantidad de votación. La propuesta presentada por el grupo de estudiantes observado fue finalmente la ganadora.

\section{Resultados y análisis}

En este punto se presentan los primeros procesamientos respecto del material proveniente del trabajo de campo realizado. Estos permiten ofrecer algunas notas respecto de las formas en las que los estudiantes participan en sus clases, así como las forman en que ellos significan esos modos de participar. Una de las primeras cuestiones que aparece es que los estudiantes no participan de la misma manera en todos los espacios que ofrece el contexto escolar. Esto lleva a pensar que hay algo de los contextos que parece ponerse en juego en cada una de las clases, y cuya variación provocaría comportamientos diferenciales en el estudiantado. Entonces, para comprender la participación, se debe atender a aquello que se ofrece en cada situación y contexto.

\subsection{Ser o estar: La participación de los estudiantes en el salón de clases}

Pensar en la variación que se produce respecto de las formas en que los estudiantes participan de sus clases, desde la perspectiva situacional o contextualista del aprendizaje que se emplea aquí, conlleva necesariamente la comprensión del contexto o situación donde acontece. Esto significa que la unidad de análisis, para entender las variaciones posibles, no sería el sujeto, sino la relación entre sujeto y contexto, como algo inherente. Según Rodrigo (1994), el contexto se considera "como algo inseparable de las acciones humanas, y no como una mera variable externa que influye en ellas" (p. 31). En otros términos, la participación de los estudiantes parece variar de acuerdo con las características que adoptan las propuestas curriculares que ofrecen los docentes, así como el propio estilo o modelo docente puesto en juego.

La consideración del contexto, ha sido ampliamente abordada por múltiples estudiosos del tema. Respecto de esto Bárbara Rogoff formula la categoría de actividad o evento. Dicha formulación habilita modificar la mirada tradicional que escinde al individuo del entorno físico o cultural. Las categorías de actividad o evento son propuestas por la autora, como formas posibles de pensar el aprendizaje y el desarrollo consisten en lo siguiente: 
(...) En lugar de analizar la posesión de una idea o una capacidad se centra en el análisis de los cambios activos que se producen en un evento en curso y en los que las personas participan [...] Los eventos o metas se organizan en torno a metas que le dan sentido [...] los procesos mentales no pueden analizarse con independencia de la meta y de las acciones prácticas e interpersonales que intervienen para lograrla [...] el pensamiento no puede surgir como algo significativamente separado de la acción, las circunstancias y la meta [...] La multiplicidad es algo esencial en el proceso de desarrollo. Más que asumir que el desarrollo avanza hacia un solo punto final universal. (Rogoff, 1990, citado en Baquero y Limón, 2011, p.167)

Tres son las dimensiones que, conectadas entre sí, componen la categoría de actividad, y que permiten analizarla: apprenticeship, participación guiada y la apropiación participativa. Esta última dimensión se encuentra emparentada con la idea de que las prácticas educativas son de alguna manera comunidades de aprendizaje. La participación de los sujetos en el seno de una determinada comunidad implicaría, de algún modo, la conversión de los mismos en otros distintos: Respecto de estos, señala Rogoff "al modo en que los individuos se transforman a través de su implicación en una u otra actividad, preparándose en el proceso para futuras participaciones en actividades relacionadas [...] se trata de un proceso de reconversión, más que de adquisición” (Rogoff, 1997, p. 3). Desde la perspectiva de esta autora, los sujetos no toman parte de una situación, sino que son parte de ella.

En función de lo señalado, y teniendo en cuenta las variaciones que se encontraban respecto de las formas de participar por parte de los sujetos en los diversos espacios de la propuesta escolar, se puede decir que la escuela no sería un contexto, sino varios contextos y situaciones desarrollándose en un mismo edificio escolar.

Ahora ¿Qué piensan los profesores respecto de la participación de sus estudiantes? Para algunos de ellos, la participación varía según los grupos de estudiantes. Para otros, hablar, tomar la palabra aparece vinculado como la acción de participar. Contrariamente, para otros docentes hablar aparece como signo de desorden, como desvinculado de las tareas escolares. Respecto de esto señalaba una docente "Ellos son muy participativos. Hacen la tarea y después se ponen a hablar. Los otros grupos no hacen nada, solo hablan". Finalmente, algunos docentes hablan de participación vinculada a lo político, y lo político aparece como algo malo. La paradoja es que, según las observaciones de clase, los 
docentes que piensan en lo político como algo malo, son docentes que enseñan sobre política y participación de modo teórico mientras les piden a sus estudiantes que hagan silencio, que escuchen las definiciones, que las copien en sus carpetas.

Es variopinto el abanico de concepciones que circula respecto de las formas de pensar la participación por parte de las personas docentes. Es variopinta la forma en que promueven o no la participación en sus clases también. El abanico de propuestas observadas abarcaba desde docentes muy concentrados en la mantención de la disciplina y el orden en el aula, la enseñanza mecánica y memorística, docentes con propuestas un tanto más abiertas aunque con actividades que promovían la infantilización de los jóvenes (tales como completar palabras en fotocopias, etc.), hasta propuestas donde los estudiantes tenían una participación muy activa (como elegir un tema de investigación y desarrollarlo). Los jóvenes estudiantes parecían leer con mucha claridad qué es lo que esperaban sus docentes de ellos, y comportarse en función de eso.

Como se describirá más adelante algunos estudiantes señalarán que participar en sentirse inmerso en las actividades que se proponen, tomados por la situación, mientras otros, ante actividades que promueven una pronunciada autonomía, comenzaban a preguntar cuándo se iba a comenzar con la clase verdaderamente, demostrando en algún punto, que las propuestas donde se debe debatir, pensar, reflexionar, para ellos, se desvincula de la tarea escolar, pierde el sentido hacerlas.

Por todo lo antedicho, es posible pensar que las características que adopten las diversas actividades propuestas por los docentes, vinculadas con el modo de participar de los estudiantes, pueden alterar las formas en que los jóvenes se apropien, participen y signifiquen las prácticas que en la escuela tienen lugar.

\subsection{De la calle a la escuela: devenir estudiantes}

Para Packer y Goicoechea (2000) el aprendizaje implica la conversión de los sujetos en miembros de una comunidad, y la construcción, allí, de diversos niveles de conocimiento a partir de su pericia como estudiantes. Para este autor, el aprendizaje también implica la necesidad de que los sujetos devenidos en estudiantes tomen partido respecto de la cultura de su propia comunidad. A decir de Packer y Goicoechea (2000) "El aprendizaje conlleva la transformación tanto de la persona como del mundo social" (p. 1) y desde esa premisa propone pensar en las tensiones que pueden producirse en el proceso en que el sujeto lucha por volver propia la interpretación implícita que circula en el contexto escolar respecto de lo 
que significa ser un "estudiante". Es por ello, señalan Packer y Goicoechea (2000), -quienes discuten con las perspectivas constructivistas, el aprendizaje conlleva cambios no solo epistemológicos en los sujetos, sino fundamentalmente ontológicos.

Según el autor, la llegada del niño o joven a la escuela implicaría, tal como se señaló anteriormente, la conversión de este sujeto en estudiante, y esto implica, en sí mismo, una transformación ontológica. Respecto de este punto sostiene: "El nuevo tipo de individuo no reemplaza al antiguo - los niños regresan a casa al final de cada día - pero tampoco es simplemente añadido. El niño asume diferentes modos de subjetividad en los dos contextos diferentes" (Packer y Goicoechea, 2000, p. 20).

Desde la posición de estudiante, los sujetos pasan a estar sometidos a las reglas de la comunidad escolar. Según Packer y Goicoechea (2000) "Las reglas del salón de clase son típicamente presentadas como un orden disciplinario objetivo de acuerdo con las que se espera que los niños actúen" (p. 20). Asimismo, se señala que las relaciones impersonales y las abstracciones son sostenidas por las prácticas que acontecen dentro de la comunidad del salón de clases. De esta manera, son constantemente reproducidas en el marco de las prácticas, aunque también es posible que muchos de los sujetos las rechacen por diversas razones de la comunidad del salón. Respecto de este punto, Packer y Goicoechea (2000) insisten en que el salón de clases es un sitio de producción cultural activa y no solo de intercambio. Allí, los estudiantes no son meros receptores pasivos, sino que devienen en participantes activos de la cultura del aula, quienes comprometidos con la reproducción de la comunidad pueden, incluso, transformarla. En este sentido, señala que los estudiantes pueden estar alineados a favor o en contra de la autoridad del maestro.

Para Packer y Goicochea (2000), el aprendizaje en la escuela implica la apropiación no solo de unos ciertos contenidos, sino también de unas ciertas formas de estar y de ser en el salón de clases. Según Michell Foucault, el salón de clases es una "matriz disciplinaria", según Jaques Lacan, es un "orden simbólico". De este modo, al incluirse los estudiantes en el salón de clases, esto no solo estaría generando conocimientos y habilidades, sino que conlleva en sí mismo un trabajo ontológico: la transformación de la persona humana (Packer y Greco-Brooks, 1999).

Respecto de la participación de los estudiantes en sus actividades de aprendizaje, señalan Packer y Goicoechea que ellos: 
[...] Pueden aceptar o rechazar los costos de la participación en la comunidad, adoptar o buscar evitar, o superar, la escisión que se les demanda. (...) El compromiso significa una actitud de alineación con y aceptación de los términos de la posición de uno en el salón de clase: la resistencia significa una actitud de oposición a, y rechazo de la autoridad del maestro y de la posición de estudiante, a menudo de una manera que en cambio busca la posición frente a los pares - la segunda fuente de reconocimiento en el salón de clase. (2000, p. 22)

De acuerdo con la perspectiva propuesta por Packer y Goicoechea (2000) respecto de la participación, y en virtud del trabajo de campo realizado, es posible decir que gran parte de lo que hacían los estudiantes parecía estar relacionado con una cierta necesidad de ellos por "cumplir" con lo que los y las docentes pedían implícita o explícitamente para aprobar la materia en cuestión. Esto se expresa con claridad cuando varios de los estudiantes señalan que participar es "Hacer lo que pide el profesor".

De este modo, algunas de las actividades que realizaban para cumplir con lo que se esperaba de ellos/as eran: pasar al pizarrón, entregar la tarea, mantenerse sentados, hacer que el/la profesor/a vea que se está trabajando. El/la profesor/a por su parte, se encargaba de tomar nota en sus cuadernos, tanto el cumplimiento de los estudiantes respecto de las tareas pedidas, como de su comportamiento.

Esta necesidad de "cumplir" con lo que se pide, se hace especialmente evidente en los momentos de "cierre de notas", donde los estudiantes, para aprobar deben haber cumplido con lo requerido. Cada final de trimestre, el profesor/a nombraba a los/las estudiantes dando cuenta de su situación. En el caso del/la estudiante que no cumpliera con lo requerido, los docentes podían ponerle directamente una baja nota, o bien, otorgar nuevas oportunidades para que los/as estudiantes completaran los trabajos faltantes. Esta última situación solía ser la habitual. Los estudiantes por su parte, pedían prestadas las actividades y las copiaban para entregarlas.

En el caso de que algún profesor se mostrara reticente a dar oportunidades, los estudiantes, generalmente en grupitos de dos o tres, se agolpaban alrededor del escritorio, con el objetivo de convencerlo de que lo aprobara. Esto claro, dependía de qué profesor se tratase.

Toda esta descripción, que puede ocurrir en centenares de escuelas cada día, intenta llamar la atención respecto de lo que señala Packer y Goicoechea (2000) sobre la 
participación en clase: estar en la escuela y transitar exitosamente por ella parece implicar más, conocer las reglas del espacio escolar, dentro y fuera del aula, pero también conocer aquello que el profesor espera del estudiante. Esto implica apropiarse de un modo de ser, o específicamente de hacer lo que se pide. Por supuesto que la pregunta es qué acontece con el aprendizaje. Es posible decir entonces, que la apropiación que se produce es respecto de unas ciertas reglas sociales y culturales, pero no de contenidos curriculares, estableciéndose así una relación débil con el conocimiento. En algunos casos, se produce lo que Kessler (2007) denominó escolaridad de baja intensidad, es decir, casos de estudiantes que van a la escuela y están en el aula, pero sin hacer ningún tipo de actividad.

Incluso, resulta interesante señalar que, frente a alguna propuesta alternativa de aprendizaje $^{8}$, los estudiantes preguntaban para qué era, si la actividad llevaba nota, si era obligatorio participar, hasta en algunos casos, decidían no formar parte del proyecto.

Esto podría estar demostrando que para algunos estudiantes la apropiación de la experiencia escolar estaría más relacionada con aprender ciertas reglas de la vida social del aula, más que la adquisición de los contenidos. En línea con lo que señala Packer y Goicoechea (2000), la participación tanto en los currículos formales como ocultos es un medio para lograr este fin. Un niño que rechaza la realidad de la comunidad de práctica del salón de clase, al encontrar que los costos son demasiado altos, puede parecer no estar logrando aprender cuando él o ella están buscando una base para una identidad en oposición.

Veamos en el siguiente punto, qué es lo que efectivamente dicen los estudiantes respecto de su participación en la clases.

\footnotetext{
${ }^{8}$ Se está haciendo referencia al proyecto NEPSO "Nuestra Escuela Pregunta Su Opinión", implementado por una de las profesoras del curso. Dicho proyecto tiene como objetivo promover una forma de aprendizaje donde los estudiantes junto a sus docentes escogen y desarrollan un tema de investigación mediante la utilización de la encuesta de opinión como instrumento de recolección de datos. Este proceso, intenta estimular, por un lado, que la apropiación de los aprendizajes por parte de los alumnos adquiera mayores grados de significatividad y contextualización, a la vez que estimule la posibilidad de alterar las formas tradicionales de distribución de posiciones subjetivas en que se ha organizado la experiencia escolar. La producción de conocimientos por parte de los alumnos, tanto dentro de sus escuelas como en las comunidades locales en las que habitan, o sobre la realidad social, política y económica que los circunda se constituye de esta manera en una forma de acceder a los contenidos del currículum de una forma diferente, por cuanto estas investigaciones pueden ser trabajadas articulando diversas áreas o sub áreas, diversos conocimientos, etc. En este orden de ideas, aun cuando los temas de investigación puedan variar de una experiencia a otra, la constancia está dada por la necesidad de promover el protagonismo de niños y jóvenes como autores de su propio aprendizaje sobre un universo que les es cercano y significativo, y por la posibilidad de generar una forma de trabajo cooperativo.
} 


\subsection{Participación y apropiación de la experiencia escolar: implicancias ontológicas y formas posibles}

En línea con lo planteado por Packer y Goicoechea (2000) respecto de la participación, para Bustamante Smolka (2010), la apropiación de la cultura por parte de los sujetos implicaría la producción de cambios de tipo ontológicos en los sujetos. En este sentido, señala que la producción de signos y sentidos por parte de los sujetos es inherente a la dinámica de las interrelaciones de las que participa. Interrelaciones que afectan la constitución subjetiva de los sujetos, produciendo transformaciones ontológicas. Desde esta perspectiva, apropiación deviene en una categoría relacional, por cuanto no estaría significando una posesión o la adquisición de, sino que su constitución se produciría en el seno de unas interrelaciones determinadas.

Para esta autora, la apropiación se encuentra íntimamente ligada a la significación que los sujetos construyen respecto de sus participaciones en cada una de las actividades de las que son parte. Estaría atravesada por los sentidos que los sujetos adjudican a las prácticas, por las posiciones que ocupan y por los modos de participación que practican en una actividad determinada. No obstante, Bustamante Smolka (2010) señala que la participación en una actividad determinada no implicaría, para los sujetos, la capacidad de controlar lo que allí acontece, ni de la multiplicidad de sentidos que se producen.

Tomando como referencia el planteamiento que esta autora realiza, será posible pensar en la existencia de diversas formas de participación y apropiación de una actividad e incluso de intensidad.

Por otra parte, autores como Trilla y Novella (2001) se han encargado de caracterizar la participación de los sujetos a partir de varios niveles de implicación. El nivel más complejo de participación sería la metaparticipación, por cuanto implica una reflexión del propio sujeto respecto de su participación en una actividad determinada. Una forma de participación de menor complejidad sería aquella denominada proyectiva, que si bien no implica una reflexión respecto de las formas de participación, si implicaría una participación en la toma de decisiones respecto de una actividad determinada.

La participación denominada como consultiva sería aquella donde la opinión de los participantes cobra un tanto más relevancia, aunque quienes realizan la consulta podrían tomar o no dichas opiniones o pensamientos. Finalmente, la forma simple de participación sería aquella donde los sujetos asumen lugares relacionados con la ejecución o con la 
observación de una tarea, sin haber participado de la elaboración de esta respecto de su contenido o desarrollo.

\subsection{Las voces de del estudiantado y la participación en el salón de clases: sentidos y significaciones}

Tomando como referencia las perspectivas citadas es posible advertir, en principio, que los y las estudiantes suelen mostrar diversas formas de participación según el espacio curricular al que se esté haciendo referencia, así como también una variación dentro del mismo espacio curricular, según la actividad propuesta o el momento del año del que se trate. Esto pone en tensión el concepto que señala la existencia de una "voz del estudiantado" (Rudduck y Flutter, 2007), por cuanto lo que existe es una pluralidad de voces, según los tiempos y los espacios. Esto es aún más evidente cuando se observa la existencia de conflicto de voces dentro del salón de clases, no solo entre docentes y estudiantes, sino también entre los mismos estudiantes: Voces que se imponen, silencios constantes, voces bajas, etc. Esto podría ser una muestra de la existencia de distintas formas de ser y estar en las clases, según cada estudiante. No obstante, en este punto, nos vamos a detener en las formas posibles de participación, según lo que se enuncie por parte de los mismos estudiantes.

Conforme con los estudiantes, existen actividades en las que se sienten más implicados o inmersos que en otras. Respecto de este punto, señala Belén, una estudiante del curso en cuestión, participar es "Estar presente en la clase, inmersa”. Esta estudiante hace una diferencia respecto de estar en la clase y participar en ella. Como contraejemplo de la participación en clase, describe lo que suele suceder en una de las clases a las que semanalmente asiste. Luego de señalar la rigurosidad del profesor en cuestión en términos de las reglas que impone, señala que para ella, transitar esa materia es como "Hacer un trámite (...) Lo que tenés que hacer es cumplir con lo que te pide y listo (...) Siento que no estoy en la clase (...) El profesor piensa que esto es la universidad".

En conformidad con las palabras de esta estudiante, y tomando como referencia a Rogoff, tomar parte implicaría la "inmersión/implicación del estudiante en la actividad. Es una actividad donde el sujeto se siente implicado, tomado o interpelado por la situación que se presenta" (Lucas, 2015, p. 14).

Sin embargo, la referencia a la implicación por parte de los estudiantes suele ser menor en términos de cantidad. Lo que predomina en los enunciados de los jóvenes cuando 
se les pregunta lo que entienden por participación, es la actividad de hablar. El hablar, tomar la palabra en clase, hacer uso de ella, aparece para estos jóvenes como sinónimo de participar. No obstante, en una indagación más profunda, hablar estaría significando por un lado, formas de diálogo donde los estudiantes hacen referencia al tema que se está tratando en la clase, mientras por otro, se hace referencia a aquellas "intervenciones donde algún estudiante habla, aun cuando lo que diga, no se encuentre absolutamente relacionado con el tema que se está tratando en la clase" (Lucas, 2015, p. 15). Así, fue muy común encontrar que los estudiantes califiquen como "los más participativos" a aquellos compañeros que suelen hablar mucho públicamente en la clase, o con otros compañeros, pero no sobre actividades referidas al aprendizaje. En este caso, la implicación en la actividad propuesta por el profesor puede estar o no presente, y dependerá del tipo de participación que esté protagonizando el estudiante.

En resumen, si bien gran parte de los estudiantes conciben la participación como "hablar", lo que se observa en los hechos es que cuando se habla, cuando algunos estudiantes, incluso señalados como los más "participativos" hablan, no siempre se hace en relación con la actividad propuesta, o con el tema que se está tratando en la clase.

Finalmente, habría una tercera forma de entender la participación por parte de los estudiantes - en línea con lo que se señaló respecto de las observaciones en el campo tiene que ver con cumplir con aquello que el profesor pide. Una característica de esta forma de participación no es la carencia de apropiación, sino que su existencia estaría más bien relacionada con una forma de comportamiento ligada a la expectativa del profesor/a. Lo que se busca, tal como se ha señalado anteriormente, es satisfacer las expectativas del profesor/a. Tal como se ha dicho ya, para muchos participar está relacionado con satisfacer las demandas del profesor. En algunos casos, señalados como los más extremos, algunos estudiantes decían que "En derecho, si no participas el profesor te saca del aula" (Lucas, p.14.). En este caso, varios/as estudiantes decían participar por miedo a no aprobar la materia. Participar, según un estudiante "[...] es hacer lo que el profesor te pide [...] comportándonos como soldados [...] por ahí te preguntan algo y vos tenés que contestar" (Lucas, p. 14).

Según las observaciones, en este tipo de clases no parecen existir más demandas que aquellas relacionadas con la aclaración respecto de las formas de realizar una actividad. De este modo, es posible decir que la apropiación que se produce respecto del aprendizaje, parece ser sumamente débil, pues no habría implicación subjetiva de los jóvenes 
estudiantes. Lo que se mantiene vigente es la apropiación de un conjunto de reglas, normas, y la necesidad de responder a las expectativas y pedidos implícitos y explícitos de Las personas docentes.

Ante la pregunta respecto de qué es lo que determina sus participaciones en las clases, los estudiantes dicen que no suelen participar del mismo modo en todas las clases. Señalan que participan o actúan según la forma de ser del profesor. En menor medida se señaló que lo que determina la participación en la clase es el interés que esta despierte.

\subsection{Los estudiantes y sus relaciones con la producción de los saberes}

Para concluir esta aproximación respecto de las formas de participación y apropiación de los estudiantes y de los sentidos y significaciones puestos en juego en sus escuelas, se aborda, en este punto, la pregunta acerca de los sentidos enunciados por los estudiantes respecto de su vínculo con la experiencia escolar en general. Según los aportes de Bernard Charlot (2007), se intentó distinguir entre la relación que los jóvenes mantienen con el saber, respecto de aquella que se mantiene con la escolarización en general.

Ante un contexto donde gran parte de las investigaciones se preguntan respecto de por qué se produce el denominado fracaso escolar, Charlot (2007), se anima a preguntar ¿Por qué estudiaría un estudiante? Para este autor, la pregunta acerca de por qué un estudiante se propondría estudiar, en definitiva se encuentra relacionada con los sentidos que se ponen allí en juego: a) el sentido acerca de concurrir a la escuela; b) el sentido puesto en juego en el "estudiar"; c) el sentido respecto de aprender dentro de la escuela y/o fuera de ella. Respecto de esto, señala Charlot:

[...] Para comprender las dificultades escolares del niño, no basta con preguntarse lo que no tiene y lo que no es, debemos primero y antes que nada analizar las situaciones que vive, sus prácticas (y las de aquellos que lo educan) y el modo en que las interpreta. Lo que nos lleva a estudiar su relación con el saber (y de modo más amplio «el aprender») y con la escuela (y, más ampliamente, con otros lugares de educación y de formación). (2007, p. 13)

Para este autor, habría que diferenciar entre la relación que los estudiantes mantienen con la escuela de las que mantienen con los saberes de los que allí pueden apropiarse - 0 no-. En definitiva, la diferencia está puesta en el sentido en que un sujeto determinado, un estudiante, puede adjudicarle a las mencionadas actividades. 
En este sentido, cabe mencionar la diferencia entre las formas en que los estudiantes participan en sus actividades - o no- y los sentidos puestos en juego respecto de ir a la escuela. Mientras la participación en el salón de clases puede ser escasa o nula, algunos jóvenes mencionan que van a la escuela para "Ser alguien en la vida", "Para el día de mañana tener un trabajo". Lo que se revela de este modo es que aun cuando la relación con los saberes pueda ser débil, la relación con la escuela tiene un profundo sentido subjetivo puesto en juego. Respecto de esto señala Charlot:

[...] Los propios jóvenes expresan la síntesis entre la dimensión social y singular de la existencia diciendo que lo esencial es para ellos "llegar a ser alguien", a través de la escuela o de otros medios, inclusive, a veces, ilegales. Lo esencial, es el proyecto de vida, el proyecto de sí, la esperanza de ver reconocido su valor por los otros...y por sí mismo [...] Ser joven, es confrontarse al desafío, y a la necesidad de transformarse en alguien, a la vez semejante a los demás y diferente a ellos, apropiándose del mundo que ya está allí. (2007, p. 15)

En un contexto donde la obligatoriedad de la escuela secundaria ha devenido mediante ley en obligatoria, y donde los índices muestran con claridad que hay más jóvenes que están dentro de la escuela, la pregunta por la relación con los saberes, y en definitiva, por la calidad educativa misma, se vuelve absolutamente imprescindible.

\subsection{Conclusiones}

La necesidad de promover actividades donde la niñez y la juventud devengan en sujetos de palabra, de voz, es - y viene siendo desde hace décadas - para los educadores, un objetivo, una utopía, quizás algunas veces colectiva. En este sentido, es posible verificar la existencia de diversas experiencias que logran una irrupción un tanto más legítima de las voces de los estudiantes, modificaciones en las formas tradicionales de relacionarse entre docentes y estudiantes, mayores grados de apropiación de las experiencias ofrecidas por la escuela, entre otros logros de la experiencia educativa.

No obstante, tomando como referencia las voces de los estudiantes en esta investigación, así como la propia experiencia subjetiva del trabajo de campo, gran parte del tiempo escolar de los estudiantes aparece destinado a cumplir con las expectativas de los profesores como una forma posible de obtener la aprobación de las materias. La apropiación 
de aprendizajes y la implicación subjetiva en las actividades propuestas aparecen en los relatos con marcas débiles, algunas veces por horas inexistentes.

Esta presentación omite mostrar dentro de la experiencia analizada, la existencia de proyectos, así como de profesores que buscaban ofrecer actividades que interpelaban a los estudiantes de diversas formas. Solo como ejemplo, podemos señalar que durante la realización del trabajo de campo, se realizó, desde la escuela, la elección de centro de estudiantes - en ese momento esto era parte de una política educativa provincial —, que fue ganada por una propuesta presentada desde el curso observado. Asimismo, una de las profesoras - con el aval de la dirección de la escuela - llevó adelante el Proyecto NEPSO. Algunos de los profesores, por su parte hacen verdaderos esfuerzos por hacer que sus estudiantes aprendan De esto que se ofrecía como experiencias "alternativas", solo se apropiaba una parte de los estudiantes, mientras otros no mostraban interés, o no encontraban el sentido de participar de estas actividades. Algunas de las frases que se decían al respecto eran “¿Esto para qué sirve?” o “¿Esto va con nota?”.

La participación para este grupo de estudiantes - o para la mayor parte de ellos significa cumplir con las expectativas de la persona docente, con las normas y reglas escolares. Sin embargo, si bien la relación con el aprendizaje o con los saberes aparece como débil, ir a la escuela tiene una significación muy profunda y relacionada con sus trayectorias vitales a futuro. Ir a la escuela implica la posibilidad de ser alguien mañana, de tener un mañana.

Los resultados que se desprenden de la investigación realizada pretenden colaborar en la discusión respecto de la necesidad de aumentar la participación de niños y jóvenes en las escuelas. Como podrá observase, mientras la implementación de proyectos y programas no vayan de la mano con la reflexión acerca de los modos de promover esa participación desde los adultos de la institución escolar, probablemente se siga reproduciendo aquello que se pretende combatir.

\section{Referencias}

Baquero, Ricardo y Lucas, Julia. (2015). Las paradojas de un alumno autónomo. Sobre las formas de apropiación de una experiencia educativa en escuelas de nivel medio del Área Metropolitana de Buenos Aires. En Bento Selau y Rafael Fonseca de Castro (Eds.), Cultural Historical Theory: Educational Research in Different Context, (pp. 39-60). Porto Alegre: EDIPUCRS.

Baquero, Ricardo. (2001). La educabilidad bajo sospecha. Cuadernos de Pedagogía Rosario 4(9), 71-85. 
Baquero, Ricardo y Limón, Margarita. (2011). Introducción a la psicología del aprendizaje escolar (2da edición). Bernal: Universidad Nacional de Quilmes

Batallán, Graciela y Campanini, Silvana. (2008). La participación política de niñ@s y jóvenesadolescentes: Contribución al debate sobre la democratización de la escuela. Cuadernos de antropología social, (28), 85-106. Recuperado de http://www.scielo.org.ar/scielo.php?script=sci arttext\&pid=S1850-

275X2008000200005\&lng=es\&tlng=es

Charlot, Bernard. (2007). Juventud y educación: Aproximaciones filosóficas y sociológicas. En Adriana Marrero (Comp.), Todas las escuelas, la escuela (pp. 87-106). Valencia: Germania.

Bustamante Smolka, Ana Luiza. (2010). Lo (im)propio y lo (im)pertinente en la apropiación de las prácticas sociales. En Nora Elichiry (Comp.), Aprendizaje y contexto: contribuciones para un debate (pp. 41-60). Bs As.: Manantial.

Kaplún, Mario. (2004). Los jóvenes, múltiples miradas. Neuquén, Argentina: Universidad Nacional del Comahue.

Kessler, Gabriel. (2007). Escuela y delito juvenil. La experiencia educativa de jóvenes en conflicto con la ley. Revista Mexicana de Investigación Educativa, 12(032), 283-303.

Kravetz, Silvia y Castro, Alejandra. (2015). La inclusión educativa en la escuela secundaria de la Provincia de Córdoba. Reflexiones desde la investigación. En Daniel Pinkatz (Comp.), La investigación sobre educación secundaria en la Argentina en la última década (pp. 46-60). FLACSO, Buenos Aires, Argentina

Lucas, Julia. (noviembre, 2015). Participar en la escuela ¿Ser o estar? Primeras aproximaciones a un estudio sobre la participación de los jóvenes estudiantes en sus experiencias escolares. Ponencia presentada en la V Jornadas de Becarios y Tesistas 2015 de la Universidad Nacional de Quilmes, Quilmes, Arguentina. Recuperado de http://sociales.unq.edu.ar/wp-content/uploads/byt2015/eje09.html

Packer, Martin y Goicoechea, Jessie. (2000). Teorías socioculturales y constructivistas del aprendizaje: ontología, no solamente epistemología. Recuperado de http://www.psicologiacultural.org/Pdfs/Traducciones/Teorias\%20socioculturales $\% 20 \mathrm{y} \%$ 20constructivistas.pdf

Packer, Martin y Greco-Brooks, David. (1999). School as a site for the productions of persons. Journal of Constuctivist Psyhcology, 12(2), 133-151. Jacqueline Garavito y María Cristina Tenorio (Trad.). Grupo de investigación Cultura y Desarrollo Humano, Universidad del Valle, Cali.

Redondo, Patricia. (2004). Escuelas y pobreza. Entre el desasosiego y la obstinación. Buenos Aires: Paidós.

Rodrigo, María José. (1994). Contexto y desarrollo social. Madrid: Síntesis. 
Rogoff, Bárbara. (1997). Los tres planos de la actividad sociocultural: apropiación participativa, participación guiada y aprendizaje. Recuperado de http://www.terras.edu.ar/biblioteca/6/TA Rogoff Unidad 5.pdf

Rudduck, Jean y Flutter, Julia. (2007) Cómo mejorar tu centro escolar dando la voz al alumnado. Madrid: Editorial Morata.

Trilla, Jaume y Novella, Ana. (2001). Educación y participación social de la infancia. Revista Iberoamericana de Educación, 26, 137-164. 